UW Biostatistics Working Paper Series

$10-22-2007$

\title{
Nonparametric and Semiparametric Group Sequential Methods for Comparing Accuracy of Diagnostic Tests
}

\author{
Liansheng Tang \\ George Mason University, ltang1@gmu.edu
}

Scott S. Emerson

University of Washington, semerson@u.washington.edu

Xiao-Hua Zhou

University of Washington, azhou@u.washington.edu

\section{Suggested Citation}

Tang, Liansheng; Emerson, Scott S.; and Zhou, Xiao-Hua, "Nonparametric and Semiparametric Group Sequential Methods for Comparing Accuracy of Diagnostic Tests" (October 2007). UW Biostatistics Working Paper Series. Working Paper 316.

http://biostats.bepress.com/uwbiostat/paper316 


\section{Introduction}

Medical tests for diagnosis of disease are often based on the comparison of some biologic measurement to some threshold. In evaluating the utility of a particular test and threshold, it is common to consider its sensitivity (the probability that a truly diseased patient has a "positive" test exceeding the threshold) and the specificity (the probability that a truly healthy patient has a "negative" test in which the measurement does not exceed the threshold). However, the optimal threshold for any such biologic measurement depends on the prevalence of disease in the screened population and costs associated with incorrect diagnoses. It is thus common to compare two diagnostic tests with respect to their receiver operating characteristic (ROC) curves: plots of the relationship between the true positive rate (the sensitivity) and the false positive rate (FPR) (one minus the specificity) as the choice of threshold varies. When the ROC curve for one diagnostic test is uniformly greater than the ROC curve for another diagnostic test, the use of the first test will tend to engender lower costs from misdiagnosis no matter the magnitude of costs assigned to each type of diagnostic error and no matter the prevalence of disease in the screened population. As a measure of the tendency for the ROC curve for one test to dominate another in this manner, it is common for investigators to consider the area under each ROC curve (AUC) Diagnostic tests with the larger AUC are deemed superior. However, when the ROC curve of a test does not dominate that of another test, the AUC may not be a good measure to use for the comparison of two tests. Other measures, such as the partial area under the ROC curve within some range of acceptable specificity or sensitivity (pAUC), or some weighted average of the ROC curve (wAUC), should be used instead (see Zhou et al., 2002 for a more complete discussion).

Scientific studies designed to compare the utility of two diagnostic tests will typically use samples of diseased and healthy subjects as defined by some gold standard. Statistical analysis of the resulting data will focus on whether any difference in AUC (pAUC, or wAUC) 
is statistically significant. Statistical methods for comparing ROC curves might be parametric (e.g., the binormal model of Dorfman and Alf, 1969), semiparametric (Cai and Pepe, 2002), or nonparametric (Hanley and McNeil, 1982; Wieand et al., 1989). Molodianovitch, et al. (2005) provided a comprehensive study on nonparametric approaches for comparing AUCs.

In scientific studies that evaluate diagnostic tests, human experimentation raises issues related to ethics and efficiency. Interim analyses conducted at one or more times during the accrual of data in such studies can greatly improve the ability to address those ethical and efficiency issues. Such group sequential monitoring of clinical trials is commonplace, but the use of sequential sampling when evaluating diagnostic tests has not received much attention to date. Mazumdar \& Liu (2003) provided a parametric sequential method for testing the equality of two AUCs when the observations in the healthy and diseased populations follow normal distributions (the "binormal model"). Zhou et al. (2007) proposed a nonparametric method for sequentially comparing AUCs. Mazumdar (2004) provided a general guideline for performing sequential tests for diagnostic accuracy studies based on AUCs.

Sequential tests are particularly valuable for comparative diagnostic trials in medical imaging modalities. Commonly used imaging procedures include Computed Tomography (CT) and Positron Emission Tomography (PET). However, CT scanners expose the subjects to ionizing radiation from a series of X-rays. In PET, subjects have to undergo the injection of radioactive isotopes in order for their regions of interest to be measured. Unnecessarily exposing the subjects in the scientific study to an inferior diagnostic procedure is clearly undesirable. Furthermore, unnecessarily delaying the identification of a beneficial diagnostic test does a disservice to those patients who are not participating in the scientific study, but would benefit from more accurate diagnosis. As both PET and CT are expensive procedures costing as high as thousands of dollars per subject, unnecessarily prolonging a study also diverts resources from better uses. 
Wieand, Gail, James and \& James (1989) introduced wAUC estimators and the subsequent WGJJ statistic for comparing two ROC curves is the difference between two wAUC estimators. The contribution made by this article is that we show a sequentially computed modification of this WGJJ statistic has approximately uncorrelated increments covariance structure by deriving its asymptotic properties using a basic theorem in empirical process theory, therefore, allowing the use of all popular group sequential methods introduced in Jennison \& Turnbull (2000). One advantage of wAUC estimators over parametric AUC estimators in Mazumdar \& Liu (2003) is that the wAUC estimator is distribution-free and includes a large family of statistics in diagnostic tests, such as non-parametric estimators of AUC, partial AUC, and the sensitivity at a specified specificity. The wAUC estimator also includes the AUC estimator in Zhou et al. (2007) as a special case.

The paper is organized as follows. In Section 2 we show that after a specific transformation the WGJJ statistic is a Brownian motion process as information time grows, therefore, it can be applied in sequential tests. In Section 3 we describe the design and monitoring procedure for comparing the wAUC's in group sequential designs. In Section 4 we describe the use in ROC curve analysis of a sequential semiparametric estimator based on proportional hazard models. In Section 5 we carry out simulations to investigate the efficiency of these estimators under various settings. The nonparametric method is illustrated in Section 6 in the setting of lung cancer diagnosis and some discussion is presented in Section 7.

\section{Asymptotic distribution of sequential WGJJ statistic}

Suppose we have measurements from two diagnostic tests on $m$ diseased subjects and $n$ healthy subjects, where all subjects are totally independent. Denote the measurements from test $\ell(\ell=1,2)$ on the $i$ th diseased subject as $X_{\ell i}$ and the corresponding measurements on the $j$ th healthy subject as $Y_{\ell j}$. Define joint cumulative survivor distribution functions $\left(X_{1 i}, X_{2 i}\right) \sim \bar{F}\left(x_{1}, x_{2}\right)$ for the diseased population's measurements and $\left(Y_{1 j}, Y_{2 j}\right) \sim \bar{G}\left(y_{1}, y_{2}\right)$ 
for the healthy population's measurements. Further define marginal survivor distributions $X_{\ell i} \sim \bar{F}_{\ell}(x)$ and $Y_{\ell j} \sim \bar{G}_{\ell}(y)$.

Without loss of generality, we assume that measurements tend to be larger for diseased subjects than for healthy subjects. A receiver operating characteristic (ROC) curve for the $\ell$ th test can be expressed as a plot of sensitivity $\left(\operatorname{Pr}\left(X_{\ell i}>c\right)=\bar{F}_{\ell}(c)\right)$ versus false positive rate (FPR), or 1 minus the specificity $\left(\operatorname{Pr}\left(Y_{\ell j}>c\right)=\bar{G}_{\ell}(c)\right)$ as the threshold $c$ varies over the real numbers. Equivalently, we can define the ROC curve for test $\ell$ as $R O C_{\ell}(u)=\bar{F}_{\ell}\left(\bar{G}_{\ell}^{-1}(u)\right)$, where $0 \leqslant u \leqslant 1$, noting that in this parameterization $u$ corresponds to the FPR.

Wieand et al. (1989) proposed comparing two ROC curves on the basis of the weighted area under the ROC curve $\Omega_{\ell}=\int_{0}^{1}\left[\bar{F}_{\ell}\left(\bar{G}_{\ell}^{-1}(u)\right)\right] d W(u)$, with a probability measure $W(u)$ defined on the FPR, $u$, for $u \in(0,1)$. Included in this class of accuracy measures are the area under the curve (AUC) (when $W(u)=u$ for $0<u<1$ ), the partial area under the curve (pAUC) between FPRs $u_{1}$ and $u_{2}$ (when $W(u)=u$ for $0<u_{1} \leqslant u \leqslant u_{2} \leqslant 1$ ), and the sensitivity at a given level of specificity $u_{0}$ (when $W(u)$ is a point mass at $\left.u_{0}\right)$.

In particular, Wieand et al. (1989) considered a nonparametric estimator based on empirical survivor distribution functions $\hat{\bar{F}}_{\ell}(x)$ and $\hat{\bar{G}}_{\ell}(y)$. Two diagnostic tests are then compared using the difference $\Delta$ in two wAUC estimators as estimated by

$$
\hat{\Delta}=\hat{\Omega}_{1}-\hat{\Omega}_{2}=\int_{0}^{1}\left\{\hat{\bar{F}}_{1}\left(\hat{\bar{G}}_{1}^{-1}(u)\right)\right\} d W(u)-\int_{0}^{1}\left\{\hat{\bar{F}}_{2}\left(\hat{\bar{G}}_{2}^{-1}(u)\right)\right\} d W(u)
$$

In Wieand et al. (1989)'s paper, they derived the asymptotic property of $\hat{\Delta}$. However, their proof was complicated. The use of modern empirical theory can greatly simplify the proof. Here we re-derive the asymptotic distribution of $\hat{\Delta}$ in Theorem 1 by applying Lemma 3.9.27 of Van der Vaart \& Wellner (1996) to the derivative of the functional composition, because the estimator $\hat{\Omega}_{\ell}$ can be written as the sum of two independent Brownian bridges. The proof of Theorem 1 is provided in the Appendix.

THEOREM 1. Under mild regularity conditions, when $m / n \rightarrow \lambda<\infty$ as $m, n \rightarrow \infty$, the 
difference $\hat{\Delta}=\hat{\Omega}_{1}-\hat{\Omega}_{2}$ satisfies

$$
\sqrt{m}(\hat{\Delta}-\Delta) \stackrel{\mathcal{D}}{\rightarrow} \mathcal{N}\left(0, v_{X}+\lambda v_{Y}\right)
$$

where $v_{X}$ and $v_{Y}$ are given in the Appendix.

The approximate variance $\sigma_{\Delta}^{2}$ of $\hat{\Delta}$ is then $\sigma_{\Delta}^{2}=v_{X} / m+v_{Y} / n$ and can be estimated by substituting the corresponding empirical estimates in $v_{X}$ and $v_{Y}$ in Equation (A.1) in the Appendix. Using the above distributional theory, we can make statistical inference about $\Delta$ by using the nonparametric estimator $\hat{\Delta}$ with its approximately (large sample) normal distribution, $\mathcal{N}\left(\Delta, \sigma_{\Delta}^{2}\right)$. In particular, hypothesis tests of $H_{0}: \Delta=\Delta_{0}$ can be based on the normalized statistic $Z=\left(\hat{\Delta}-\Delta_{0}\right) / \hat{\sigma}_{\Delta}$. In the presence of large sample sizes and the absence of early stopping, the Z statistic has the approximately standard normal distribution under $H_{0}$. The use of this statistic in the group sequential setting is described in the next section.

\section{Use of the WGJJ statistic under group sequential sampling}

\subsection{Stopping Rules}

We consider now a group sequential sampling plan involving up to $J$ analyses of the accruing data. At the time of the $j$ th analysis, we have diagnostic test data available on the first $m_{j}$ diseased subjects and the first $n_{j}$ healthy subjects. From these data available at the $j$ th analysis, we compute for the $\ell$ th diagnostic test the empirical survivor distribution functions $\hat{\bar{F}}_{\ell j}(x)$ and $\hat{\bar{G}}_{\ell j}$ and wAUC estimators $\hat{\Omega}_{\ell j}$. These interim estimates are then used to compare

ROC curves using interim contrast $\hat{\Delta}_{j}$, its standard error $\sigma_{\Delta j}$, and the interim normalized statistic $Z_{j}=\hat{\Delta}_{j} / \sigma_{\Delta j}$.

We consider a group sequential sampling plan defined by up to four boundaries $-\infty \leqslant$ $a_{j} \leqslant b_{j} \leqslant c_{j} \leqslant d_{j} \leqslant \infty$ at each of the $J$ analyses. In order to uniquely define a stopping rule, we demand for $j<J$ that either $a_{j}<b_{j}$ or $c_{j}<d_{j}$ (or both) and that at least one of the four boundaries are finite. It is also typical that we obtain termination with a finite sample 
size by ensuring that $a_{J}=b_{J}$ and $c_{J}=d_{J}$ for some finite (but possibly random) choices of $J, m_{J}$, and $n_{J}$.

Sequential sampling proceeds by starting at analysis $j=1$. At the $j$ th analysis, measurements on the first $m_{j}$ diseased subjects and $n_{j}$ healthy subjects are used to compute the interim statistic $Z_{j}$. If $Z_{j} \leqslant a_{j}, b_{j}<Z_{j}<c_{j}$, or $Z_{j} \geqslant d_{j}$, the study is stopped without accruing more subjects. Otherwise, the study accrues sufficient subjects to be able to proceed to analysis $j+1$. We define group sequential statistic $(\tilde{M}, Z)$ by $\tilde{M}=\min \left\{1 \leqslant j \leqslant J: Z_{j} \notin\right.$ $\left.\left(a_{j}, b_{j}\right] \cup\left[c_{j}, d_{j}\right)\right\}$ and $Z=Z_{\tilde{M}}$. In the setting of comparing ROC curves, we would most often decide that diagnostic test 1 is superior, approximately equivalent, or inferior to diagnostic test 2 according to whether $Z \geqslant d_{\tilde{M}}, b_{\tilde{M}}<Z<c_{\tilde{M}}$, or $Z \leqslant a_{\tilde{M}}$, respectively.

When frequentist statistical inference is the ultimate goal, it is common to ensure that the experimentwise error is controlled at a desired level (e.g., in a two sided hypothesis test choose stopping boundaries to ensure $\operatorname{Pr}\left(b_{\tilde{M}}<Z<c_{\tilde{M}} \mid H_{0}\right)=1-\alpha$ for a desired type I error $\alpha$ ). The dimensionality of the boundary space is typically reduced through the use of a boundary shape function which defines a relationship between the exact value of the stopping boundaries $a_{j}, b_{j}, c_{j}, d_{j}$ and the statistical information available at the $j$ th analysis (which is typically $1 / \sigma_{\Delta j}^{2}$ in the case of an approximately normally distributed statistic). For purposes of sample size calculation, the boundary shape function typically specified in terms of the proportion $\tau_{j}$ of maximal statistical information available at each analysis (in the case of approximately normally distributed statistics $\left.\tau_{j}=\sigma_{\Delta j}^{2} / \sigma_{\Delta j}^{2}\right)$. Commonly cited boundary shape functions include the O'Brien-Fleming, the triangular test boundary, and the Pocock boundaries (see Jennison \& Turnbull, 2000).

The statistical literature is replete with alternative strategies for choosing stopping boundaries appropriate for particular scientific and statistical settings (see Jennison \& Turnbull, 2000; Emerson, Kittelson, \& Gillen, 2007a, b). Almost all of that statistical literature, 
and all three of the commercially available statistical software capable of implementing the general methods (S+SeqTrial, PEST, EaSt), presume a particular covariance structure for the statistics $Z_{1}, Z_{2}, \ldots, Z_{J}$. In this covariance structure, the statistical information accrued between two successive analyses is independent of all prior information accrued, and it is thus commonly referred to as an "independent increment" covariance structure (Jennison \& Turnbull, 2000, Chapter 11).

The use of the WGJJ statistic in the sequential comparison of ROC curves is greatly facilitated by showing that the statistic has an "independent increment" structure. We let $I_{j}$ denote the statistical information at the $j$ th analysis, and $\tau_{j}=I_{j} / I_{J}$ denote the proportion of maximal information as before. Define $B\left(\tau_{j}\right)=\sqrt{\tau_{j} I_{j}} \hat{\Delta}_{j}$, which is an asymptotically unbiased estimator for $\sqrt{\tau_{j} I_{j}} \Delta=\tau_{j} \sqrt{I_{J}} \Delta=$ with asymptotic variance $\operatorname{var}\left(B\left(\tau_{j}\right)\right)=\tau_{j}$.

THEOREM 2. For $j<k, \operatorname{cov}\left(B\left(\tau_{j}\right), B\left(\tau_{k}\right)\right)=\tau_{j}$.

The proof is provided in the Appendix. Thus $B\left(\tau_{j}\right)$ behaves asymptotically like a Brownian motion process with a drift parameter $\theta$, where $\theta=\Delta \sqrt{I_{J}}$. The WGJJ estimator can then be readily accommodated by standard group sequential software.

\subsection{Sample size determination}

In a wide variety of statistical models, the maximal number $\tilde{N}_{J}$ of sampling units needed is estimated by $\tilde{N}_{J}=\delta_{\alpha \beta}^{2} V / \Delta_{1}^{2}$, where $1 / V$ is the (average) statistical information contributed by a single sampling unit, $\Delta_{1}$ is the difference between $\Omega_{\ell}$ 's under the alternative hypothesis to be detected with statistical power $1-\beta$ in a level $\alpha$ hypothesis test, and $\delta_{\alpha \beta}$ is the design alternative in some standardized version of the test. For instance, in a fixed sample (no interim analyses, $J=1$ ) two-sided hypothesis test of the difference in weighted AUCs having equal sample sizes, $\tilde{N}_{J}$ might be the sample size to be accrued in each group, $\Delta_{1}=\Omega_{1}-\Omega_{2}$ might be the difference between group means under the design alternative, $V=\sigma_{\Delta}^{2}$ is the 
variance, and $\delta_{\alpha \beta}=z_{1-\alpha / 2}+z_{\beta}$. This same formula can be used in a group sequential test, providing that the value of $\delta_{\alpha \beta}$ specific to the selected stopping rule is used.

With pilot data, it is trivial to nonparametrically calculate the variance of the WGJJ statistic from Equation (2). However, if the pilot study is not yet available, we will nevertheless need parametric distribution assumptions to obtain the variance of the WGJJ statistic based on the formula in Equation (2). This means that we will have to guess explicit distributional models and parameters in the models. In the following context, we will discuss a method to obtain conservative sample sizes based on the conjectured values of AUCs without either having a pilot data or specifying model parameters.

We denote the WGJJ statistic for AUCs as $\Delta^{A} . \Delta^{A}$ is then the difference between two Wilcoxon statistics, $\hat{\Omega}_{1}^{A}$ and $\hat{\Omega}_{2}^{A}$. Hanley \& McNeil (1982) showed that an estimated AUC had a smaller variance under negative exponential models than under normal or gamma distributions, therefore, a more conservative sample size under negative exponential distribution. More importantly, the advantage of using negative exponential distributions rather than normal or gamma distributions is that we can derive the variance of $\hat{\Omega}_{\ell}^{A}$ solely from hypothesized values of AUCs, $\Omega_{\ell}^{A}$, without prespecified parameters. Sample sizes for two diagnostic arms can then be calculated without knowing the parameters in negative exponential distributions as stated in the following theorem.

THEOREM 3. Under mild regularity conditions, as $m / n \rightarrow \lambda$ when $m, n \rightarrow \infty$, the variance $v_{A}^{2}$ of $\Delta^{A}$ is then given by $v_{A}^{2}=\operatorname{var}\left(\hat{\Omega}_{1}^{A}\right)+\operatorname{var}\left(\hat{\Omega}_{2}^{A}\right)-2 \rho \sqrt{\operatorname{var}\left(\hat{\Omega}_{1}^{A}\right) \operatorname{var}\left(\hat{\Omega}_{2}^{A}\right)}$, where $\operatorname{var}\left(\sqrt{m} \hat{\Omega}_{\ell}^{A}\right)=\lambda Q_{1 \ell}+Q_{2 \ell}-(\lambda+1)\left(\Omega_{\ell}^{A}\right)^{2}$, with $Q_{1 \ell}=\Omega_{\ell}^{A} /\left(2-\Omega_{\ell}^{A}\right), Q_{2 \ell}=2\left(\Omega_{\ell}^{A}\right)^{2} /\left(1+\Omega_{\ell}^{A}\right)$ and $\rho$, the correlation between two AUCs.

Proof. With finite sample sizes $m$ and $n$, we have

$$
\operatorname{var}\left(\hat{\Omega}_{\ell}^{A}\right)=\left[\Omega_{\ell}^{A}\left(1-\Omega_{\ell}^{A}\right)+(m-1)\left\{Q_{1 \ell}-\left(\Omega_{\ell}^{A}\right)^{2}\right\}+(n-1)\left\{Q_{2 \ell}-\left(\Omega_{\ell}^{A}\right)^{2}\right\}\right] / m n
$$

Thus, as $m, n \rightarrow \infty$, it is true that $\operatorname{var}\left(\sqrt{m} \hat{\Omega}_{\ell}^{A}\right) \rightarrow \lambda Q_{1 \ell}+Q_{2 \ell}-(\lambda+1)\left(\Omega_{\ell}^{A}\right)^{2}$. 
Denote $\tilde{V}_{\ell}$ to be the right side of the above equation. Consider a null hypothese, $H_{0}: \Delta^{A}=0$ and the alternative two-sided hypothesis, $H_{A}: \Delta^{A} \neq 0$ with Type I error $\alpha$ and power $1-\beta$ at the conjectured AUC values, $\Omega_{1}^{A}$ and $\Omega_{2}^{A}$. Using a general formula in Zhou et al. (2002, Section 6.2), the required fixed sample sizes for the diseased and healthy subjects, denoted as $M_{f}$ and $N_{f}$ respectively, can be derived by

$$
M_{f}=\lambda N_{f}=\frac{\left(Z_{1-\alpha / 2} \sqrt{(2-2 \rho) \tilde{V}_{1}}+Z_{1-\beta} \sqrt{\tilde{V}_{1}+\tilde{V}_{2}-2 \rho \sqrt{\tilde{V}_{1} \tilde{V}_{2}}}\right)^{2}}{\left(\Omega_{1}^{A}-\Omega_{2}^{A}\right)^{2}} .
$$

A special case with $\lambda=1$ and $\rho=0$ is given in Hanley and McNeil (1982). Subsequently, the maximum sample sizes $M_{g}$ for the diseased subjects and $N_{g}$ for the healthy subjects can be derived for the O'Brien-Fleming test, the triangular test, and the Pocock test by $M_{g}=\lambda N_{g}=\delta_{\alpha \beta, g}^{2} / \delta_{\alpha \beta, f}^{2} M_{f}$, where $\delta_{\alpha \beta, g}^{2} / \delta_{\alpha \beta, f}^{2}$ is the sample size ratio between a fixed sample design and a sequential design. We can also get the maximum sample sizes $M_{e}$ and $N_{e}$ for a more flexible error spending function design (Lan \& DeMets, 1983) by $M_{e}=\lambda N_{e}=\theta_{e}^{2} / \theta_{f}^{2} M_{f}$, where $\theta_{f}$ and $\theta_{e}$ are the drift values for a fixed sample design and a group sequential design, respectively.

Not restricted to the two-sided hypothesis test mentioned above, the sample size can be determined for any stopping rule in Section 3.1. These test methods were incorporated in S+SeqTrial (2000) developed by Emerson and others. Their software provides a comprehensive tool for designing, monitoring, and analyzing clinical trials using group sequential methods. The independent increment covariance structure of the WGJJ statistic ensures its ready accommodation by the software. In S+SeqTrial, we can specify the type of group sequential design in $\mathrm{S}+\mathrm{SeqTrial}$, then plug in the conjectured value for a wAUC, with its variance, the desired power and type I error to obtain the total size without calculating both fixed sample size and the ratio $\lambda$. 


\section{Semiparametric partial AUC estimator}

When the measurements of diagnostic tests are from exponential distributions or other power-law distributions, the proportional hazard model assumption is satisfied (for a real example, see Sanchez-Marin and Padilla-Medina, 2006). We can then use a semiparametric estimator for comparing correlated AUCs under proportional hazard models. Let $Z_{\ell i}=1$ if the $i$ th subject is diseased, 0 otherwise, under the $\ell$ th diagnostic test with $\ell=1,2$. Now the $\ell$ th test of the $i$ th subject has a hazard function $\lambda_{\ell i}(t)=\lambda_{\ell 0}(t) \exp \left\{\gamma_{\ell} Z_{\ell i}\right\}$, where $\lambda_{\ell 0}(t)$ is a baseline hazard function and $\gamma_{\ell}$ 's are parameters in the Cox regression. The resulting ROC curve for the $\ell$ th test takes on the form of $R O C_{\ell}(u)=u^{\exp \left(\gamma_{\ell}\right)}$. Its AUC is given by $\Omega^{A}\left(\gamma_{\ell}\right)=1 /\left\{\exp \left(\gamma_{\ell}\right)+1\right\}$, and its partial AUC between $a$ and $b, 0<a<b \leqslant 1$, is given by $\Omega^{p A}\left(\hat{\gamma}_{\ell}\right)=\left\{b^{\exp \left(\hat{\gamma}_{\ell}\right)+1}-a^{\exp \left(\hat{\gamma}_{\ell}\right)+1}\right\} /\left\{\exp \left(\hat{\gamma}_{\ell}\right)+1\right\}$. If $0=a<b \leqslant 1$, the partial AUC is $\Omega^{p A}\left(\hat{\gamma}_{\ell}\right)=b^{\exp \left(\hat{\gamma}_{\ell}\right)+1} /\left\{\exp \left(\hat{\gamma}_{\ell}\right)+1\right\}$. The covariate-adjusted estimator $\hat{\gamma}_{\ell}$ is obtained from the marginal Cox regression model for the $\ell$ th test. The asymptotical property of $\hat{\gamma}_{\ell}$ 's is shown in Wei et al. (1989) by

$$
n^{1 / 2}\left(\hat{\gamma}_{1}-\gamma_{1}, \hat{\gamma}_{2}-\gamma_{2}\right) \stackrel{\mathcal{D}}{\rightarrow} N\left(0, v_{1} \Sigma^{\gamma} v_{2}\right)
$$

where $v_{\ell}=\operatorname{var}\left(\hat{\gamma}_{\ell}\right)$ and $\Sigma^{\gamma}$ is the correlation matrix of $n^{1 / 2}\left(\hat{\gamma}_{1}-\gamma_{1}, \hat{\gamma}_{2}-\gamma_{2}\right)$.

THEOREM 4. As $m, n \rightarrow \infty$, the estimated $A U C$ (or partial AUC) difference, $\Delta\left(\hat{\gamma}_{1}, \hat{\gamma}_{2}\right)$, satisfies

$$
n^{1 / 2}\left(\Delta\left(\hat{\gamma}_{1}, \hat{\gamma}_{2}\right)-\Delta\left(\gamma_{1}, \gamma_{2}\right)\right) \stackrel{\mathcal{D}}{\rightarrow} N\left(0, v_{p}^{2}\right)
$$

where

$$
v_{p}^{2}=\left(\begin{array}{c}
(\Omega)^{\prime}\left(\gamma_{1}\right) \\
-(\Omega)^{\prime}\left(\gamma_{2}\right)
\end{array}\right)^{\prime} \Sigma^{\gamma}\left(\begin{array}{c}
(\Omega)^{\prime}\left(\gamma_{1}\right) \\
-(\Omega)^{\prime}\left(\gamma_{2}\right)
\end{array}\right)
$$

Its proof in a general survival model framework is provided in the Appendix. Consequently, the sequential versions of our semiparametric proportional hazard AUC or pAUC estimators 
have asymptotically independent increments. Thus, it is straightforward to incorporate the semiparametric estimators in the group sequential designs. Our estimator is also easily adapted for sequentially testing survival outcome measurements from two diagnostic tests. The covariance matrix $\Sigma^{\gamma}$ between $\gamma_{\ell}$ 's can be consistently estimated using a sandwich estimator (Wei et al., 1989) by $\hat{\Sigma}^{\gamma}=n \hat{v}_{1} W_{1} W_{2}^{\prime} \hat{v}_{2}$, where $\hat{v}_{\ell}$ is the estimated variances for $\hat{\gamma}_{\ell}$, and $W_{\ell}$ is the residual vector calculated from the marginal proportional hazard model for the $\ell$ th test.

\section{Finite-sample property}

In this simulation study, we investigated the finite sample performance of the sequential WGJJ statistic and the previously described semiparametric procedure both in a fixed sample test $(J=1)$, a three-group sequential test $(J=3)$, a four-group sequential test $(J=4)$ and a five-group sequential test $(J=5)$. We also included a common binormal parametric approach for comparisons among these three methods. The null hypothesis of equal AUCs was set to be true and the nominal type I error was set to be 0.05 for two-sided tests. We simulated bivariate normal (Binorm), bivariate lognormal (Bilog) and bivariate exponential (Biexp) data as outcome measurements for two diagnostic tests. The bivariate normal models had the forms of $\left(X_{1}, X_{2}\right) \sim N\left((11,1), \Sigma_{1}\right)$ and $\left(Y_{1}, Y_{2}\right) \sim N\left((10,0), \Sigma_{2}\right)$, where

$$
\Sigma_{1}=\left(\begin{array}{cc}
1 & \sqrt{2} \rho \\
\sqrt{2} \rho & 2
\end{array}\right) \text { and } \Sigma_{2}=\left(\begin{array}{cc}
2 & \sqrt{2} \rho \\
\sqrt{2} \rho & 1
\end{array}\right) \text {, with } \rho=0.5 .
$$

The AUCs were thus the same from the formula of AUC under binormal models (Zhou et al., 2002): $A U C=\Phi\left\{\left(\mu_{1}-\mu_{0}\right) /\left(\sqrt{\sigma_{1}^{2}+\sigma_{0}^{2}}\right)\right\}$, where $\left(\mu_{1}, \sigma_{1}\right)$ and $\left(\mu_{0}, \sigma_{0}\right)$ are the normal parameters in diseased and healthy groups, respectively. The bivariate lognormal models had the forms of $\exp \left(X_{1}, X_{2}\right)$ and $\exp \left(Y_{1}, Y_{2}\right)$ for the diseased and healthy subjects, respectively. The AUCs under simulated lognormal models were also equal, since ROC curves 
are invariant to monotone transformations. Equal numbers of diseased and healthy subjects were considered in the simulation, i.e., $\mathrm{m}=\mathrm{n}=(50,100,200)$.

The bivariate exponential random variables were simulated using an algorithm in Gumbel (1960). The Gumbel's distribution had the form $H(x, y)=H_{1}(x) H_{2}(y)\left[1+4 \rho\left\{1-H_{1}(x)\right\}\{1-\right.$ $\left.\left.H_{2}(y)\right\}\right]$, where $\rho \in[-0.25,0.25]$. We set $\rho$ to be 0.25 . Bivariate exponential random variables were simulated with the marginal survival functions $\exp \left(-\beta_{\ell 1} x\right)$ and $\exp \left(-\beta_{\ell 2} y\right)$ for diseased and healthy subjects respectively, where $\ell=1,2$, denotes the types of tests. In the simulation, $\left(\beta_{11}, \beta_{12}, \beta_{21}, \beta_{22}\right)=(1,2,2,4)$. Since the AUCs under these exponential distributions are given by $\exp \left(\gamma_{\ell}\right)=\beta_{\ell 1} / \beta_{\ell 2}$, two resulting AUCs should be the same.

Under each of the above model assumptions, 1000 random variables were simulated and three methods including sequential WGJJ statistic, semiparametric method and parametric binormal method were fitted to the simulated data. The $Z$ statistics were then calculated based on estimated parameters and their variances. The rejection rates were obtained by comparing the $Z$ statistics with corresponding test boundaries under either the fixed sample design or sequential designs. Table 1 gives the rejection rates of all three methods with a nominal level 0.05 under both Pocock's (POC) and O'Brien \& Fleming's (OBF) criterions. In the fixed sample test, the WGJJ statistic gives the rejection rate close to the nominal level under all model specifications for sample sizes in both groups as small as 50, while the parametric binormal method greatly inflates rejection rates when the true underlying distribution is bivariate lognormal, and deflates rejection rates when the underlying distribution is in fact bivariate exponential. Under the setting of group sequential designs, the WGJJ statistic also give correct rejection rates regardless of underlying distribution models. When the underlying distributions are misspecified, the parametric and semiparametric methods inflate the rejection rates further compared with their fixed-sample counterparts. In addition, with proportional hazard model correctly specified, the semiparametric method performs well 
for both the fixed sample design and the sequential designs. In summary, the nonparametric approach based on the WGJJ statistic is robust to model specifications and it performs as well as parametric approach under correct model assumptions. Moreover, the nonparametric approach has excellent small-sample performance, which makes it a well-suited method for conducting group sequential diagnostic trials.

\section{[Table 1 about here.]}

\section{Examples}

\subsection{An illustration for sample size determination}

We used binormal and biexponential models to illustrate how to determine maximum sample sizes in the fixed sample design and group sequential design using the WGJJ statistic for AUC and partial AUC estimators, which are denoted as $\Delta^{A}$ and $\Delta^{p A}$, respectively. The equally-spaced symmetric two-sided error spending (Kim \& Demets, 1992) test with the error spending function $f(\tau)=\min (\alpha \tau, \alpha)$ was used with power 0.8 and type I error $\alpha=.05$.

6.1.1 Under bivariate normal model assumption. Suppose the binormal distribution of the test outcomes is given by $\left(X_{1}, X_{2}\right) \sim N\left(\left(\mu_{1}, \mu_{2}\right), \Sigma\right), \quad\left(Y_{1}, Y_{2}\right) \sim N((0,0), \Sigma)$, where covariance matrix $\Sigma$ had common variances 1 and covariances 0.5 . We let $\lambda=1$, equivalent to disease prevalence 0.5 . Since the distributions were known, we were able to obtain the exact variance of $\Delta^{A}$ or $\Delta^{p A}$ from the results in Equation (A.1) in the Appendix. Under the specified test setting, we obtained sample sizes $N_{g}$ from the results in Section 3.2. Here, the drift value $\theta_{e}=2.96$ for a sequential design and $\theta_{f}=2.80$ for a fixed sample design can be calculated using aforementioned softwares. The sample sizes $N_{f}$ for the fixed sample design can then be computed. The results for $\Delta^{A}$ are presented in Table 2 . We also found that the sample sizes given by Equation (2) under the same test setting are slightly more than those 
in Table 2. This ensures that without the pilot data or prior knowledge of the distributions, the sample size computed by Equation (2) can serve as a conservative initial guess.

To investigate how accurate these sample sizes are in maintaining the required power, we used several relatively large sample sizes in Table 2, such as size 929 (AUC 0.75 vs AUC 0.70), size 634 (AUC 0.85 vs 0.80) and size 91 (AUC 0.85 vs AUC 0.70) in a three group design. We simulated 1000 data sets under each binormal setting, and computed the number of times that the null hypothesis of equal AUCs was rejected. The resulting powers under aforementioned sample sizes were then $80.3 \%, 79.5 \%$ and $78.7 \%$, respectively. They were close to the nominal level $80 \%$.

[Table 2 about here.]

Suppose now we are interested in comparing partial AUCs for the false positive rate less than 0.6. For our range of false positive rate, it can be calculated that the partial AUC is between 0.18 and 0.6 (Zhou et al., 2002, Section 4). Table 2 also gives maximum possible sample sizes for testing the difference between partial AUCs.

These two sets of illustrations gave examples of deriving the maximum sample sizes for three-group sequential designs if the distributions of test outcomes are assumed known. In fact, given a specified power and a type I error, available softwares such as S+SeqTrial, PEST and EaSt can compute sample size ratios for Pocock, OBF, and error spending tests. If the required sample size for the fixed sample test is available, the maximum sample size for any sequential test can be derived by multiplying a specified constant ratio related to that sequential test. In the case that the models are unknown, if the pilot data is available, then the consistent estimates of $v_{X}$ and $v_{Y}$ are obtained by plugging in the empirical distributions and quantiles and substituting the empirical estimates of $r_{1}(u)$ and $r_{2}(u)$ in Equation (A.1) in the Appendix, respectively.

At the first glance of Table 2, one may notice that the maximum sample sizes of the group 
sequential test are larger than those of the fixed sample test. This is because these maximum sample sizes only occur in the worst scenario when group sequential tests are carried all the way to the end. Often sequential tests terminate earlier before the maximum number of subjects are recruited. Therefore, looking at expected sample sizes reveals the advantage of sequential trials. We calculated the expected sample sizes with the specified error spending test for the three group test. The fact that the expected sizes are about $81 \%$ of those of the fixed sample test ensures the early stopping of the group sequential design.

\subsubsection{Under bivariate exponential model assumption. A bivariate exponential distribution} in Gumbel (1960) was used to calculate the variance of the WGJJ estimator and that of the newly proposed semiparametric estimator. The Gumbel's distribution has the form $H(x, y, \rho)=H_{1}(x) H_{2}(y)\left[1+4 \rho\left(1-H_{1}(x)\right)\left(1-H_{2}(y)\right)\right]$, where $\rho \in[-0.25,0.25]$. Here $\rho$ was set to be 0.25 in this simulation study. For diseased and healthy groups, bivariate exponential random variables had marginal survival function of $\exp \left(-\beta_{\ell 1} x\right)$ and $\exp \left(-\beta_{\ell 2} y\right)$ for test 1 and 2. We let $\beta_{12}=\beta_{22}=1$. The values of $\beta_{11}$ and $\beta_{21}$ corresponded to the AUCs (or pAUCs) in Table 3 . We let $\lambda=1$ in the sample size calculation with the specified test setting. Not surprisingly, the nonparametric and semiparametric methods give the same sample sizes when comparing AUCs or pAUCs. The maximum possible sample sizes are listed in Table 3 for AUCs and partial AUCs.

[Table 3 about here.]

\subsection{Sequential lung cancer diagnostic trial}

Lung cancer is one of the most common cancers in the world and is the leading cause of cancer death in the United States. The lung cancer is categorized into two types: small cell and non-small cell. The non-small cell lung cancer is the most common type which is only curable with surgery in its early stages. Computed Tomography (CT) and Positron Emission 
Tomography (PET) are both the pre-operative scanning tests for the staging of non-small cell lung cancer. CT, a traditional diagnostic tool, does not perform well to differentiate between benign and malignant lesions. PET, a new scanning technique, provides higher resolution image with detailed view of regions of interest. But the results of PET are usually affected by muscle and inflammatory activities, which are considered to be factors of imprecision locations of abnormalities. Comparing the diagnostic performance of these two techniques is of extensive interest of radiologists (Lardinois et al., 2003; Silvestri et al., 2003). The gold standard in these tests is pathology results from biopsy specimens.

The staging accuracy of non-small cell lung cancer is usually between $52 \%$ and $85 \%$ for CT and between $81 \%$ and 96\% for PET (Lardinois et al., 2003; and Silvestri et al., 2003). Consider testing the null hypothesis of equal AUCs against the two-sided alternative with power 0.8 and significance level 0.05 . A possible initial alternative is likely to be $15 \%$ which is difference between $70 \%$ for CT and $85 \%$ for PET. If an investigator is interested in a nonparametric AUC estimator, corresponding to two-sample Wilcoxon statistics (Hanley \& McNeil, 1983), as an accuracy measure in a fixed sample lung cancer trial to compare CT and PET, the maximum sample size is 81 under power 0.8 , type I error 0.05 under the binormal assumption according to Table 2. If the outcome measurements are assumed to be from bivariate exponentials, totally 109 subjects in both arms would be required from Table 3. However, as mentioned in the Introduction, these diagnostic methods are expensive and carries some safety risks. While carrying out clinical trials to compare CT and PET, the results need to be monitored repeatedly to ensure that human subjects are not exposed to inferior scanning techniques. In negative trials that show equivalence of the accuracy of CT and PET, the trials need to be terminated early and the subjects can be switched to compare CT with other scanning techniques which involves combining CT and PET, or recently developed Magnetic Resonance Imaging technique. If PET is found to be more 
accurate than $\mathrm{CT}$ in the early interim analysis, it means that PET scan in staging the non-small cell lung cancer could be preferred over CT scan and should be performed more frequently in lung cancer diagnosis.

Suppose we want to design a three-group error spending test with the error spending function $f(\tau)=\min (\alpha \tau, \alpha)$. The equally-spaced symmetric two-sided test with significance level 0.05 and power 0.8 would need maximum possible sample sizes of 91 and 62 under binormal and biexponential assumptions, respectively, based on the results from Table 2 . In this test, the boundaries for the normalized AUC statistic, $Z_{j}$, can be calculated as $\left(c_{1}=-b_{1}=2.39, c_{2}=-b_{2}=2.29, c_{3}=-b_{3}=2.20\right)$. During the comparative diagnostic trial, $Z_{j}$ is computed at the $j$ th interim analysis and compared with these critical boundaries. A significant $Z_{j}$ gives early evidence that $\mathrm{CT}$ is different from PET, and the better imaging method should be adapted in detecting lung cancer. If there is no significance and we have not finished the trial on all patients, we will continue recruiting more patients until there is significant evidence in the next analysis. After all patients are scanned, if there is still no significant evidence to support the alternative hypothesis, a conclusion will be made that CT has the same diagnostic ability as PET, and cheaper CT scanning technique can be recommended for non-small cell lung cancer diagnosis. Since we already noted that the expected sample sizes under sequential tests would be as low as $81 \%$ of that under the fixed sample test, the early stopping on the average is ensured by group sequential tests.

AUC is an excellent accuracy measure if two ROC curves do not cross each other. However, when ROC curves cross, they may have similar AUCs but different partial AUCs over a range of specificities. Suppose a investigator is interested in whether there is a difference between partial AUCs of CT and PET over the false positive rate less than 0.6. In this trial, the WGJJ statistic then becomes the difference between the nonparametric estimators of partial AUCs over high specificities. An initial guess of partial AUCs is $40 \%$ for CT and $55 \%$ for 
PET, respectively. With power 0.8, type I error .05 and disease prevalence 0.5 , a fixed sample trial would require 48 patients under the binormal assumption and 55 patients under the exponential assumption according to Tables 2 and 3, respectively.

If a three-group error spending test is decided for the trial, the maximum possible sample sizes for both arms would be 54 and 62 under binormal and biexponential assumptions, respectively, based on the results in Tables 2 and 3. The boundaries of three stages for the normalized partial AUC differences are also $\left(c_{1}=-b_{1}=2.39, c_{2}=-b_{2}=2.29, c_{3}=\right.$ $\left.-b_{3}=2.20\right)$. Similar as comparative diagnostic trial based on AUC, the normalized test statistic $Z_{j}$ based on partial AUC is computed based on accruing patients at the $j$ th stage and corresponding decision will be based on whether $Z_{j}$ crosses the boundaries. Note the maximum possible sample sizes are smaller for the sequential trials comparing partial AUCs than those for AUCs. This is due to the fact that partial AUC estimator is less variable than AUC estimator.

To the best of our knowledge, no actual trials in diagnostic medicine have been conducted sequentially. To illustrate the details of calculations of monitoring decisions, we simulated a set of simple hypothetical outcomes from PET and CT scans in aforementioned lung cancer diagnostic trials. Suppose an investigator has decided to conduct a three-group sequential trial to compare the accuracy of CT and PET procedures. Under the binormal assumption, the maximum possible sample size was initially 91 in total based on the $80 \%$ power and 0.05 type I error. We simulated binormal data as measurements from 30 subjects at the first look. We then calculated interim contrast $\hat{\Delta}_{1}=0.0259$, its standard error $\sigma_{\Delta 1}=0.0673$, and the interim normalized statistic $Z_{1}=\hat{\Delta}_{1} / \sigma_{\Delta 1}=0.3848$. Since $Z_{1}$ fell within the boundary $\left(c_{1}=-b_{1}=2.39\right)$, we continued with the second look at 30 more simulated measurements. At the second look, we calculated interim contrast $\hat{\Delta}_{2}=0.1469$, its standard error $\sigma_{\Delta 2}=0.0534$, and the interim normalized statistic $Z_{2}=\hat{\Delta}_{2} / \sigma_{\Delta 2}=2.7510$ from all 60 subjects. Now $Z_{2}$ 
was outside the boundary $\left(c_{2}=-b_{2}=2.29\right)$, thus we stopped recruiting more subjects and came to a conclusion that PET has better accuracy to stage lung cancer than CT.

\section{Discussion}

In this article, we described that after modification the sequential WGJJ statistic behaves like the Brownian motion process, therefore, can be readily implemented using standard statistical software. The WGJJ statistic includes a large family of nonparametric estimators in comparative diagnostic tests, offering great modeling flexibility. We also proposed a semiparametric method for comparing two diagnostic tests based on multivariate proportional hazard models. With correct model specification, the semiparametric method can allow survival outcome measurements in the presence of censoring.

Calculating sample size is an important issue when performing group sequential trials. We illustrated an example of sample size determination based on binormal distribution assumptions. If a pilot study is available, sample sizes can be empirically determined from our results. Otherwise, conservative sample size can be determined with the knowledge of the AUCs and their correlation from Equation (2).

Both nonparametric AUC and partial AUC estimators, as special cases of the WGJJ statistics, were illustrated in sequential lung cancer trials for comparing the staging accuracy of non-small cell cancer. Accurate staging diagnosis could guide surgery to help prolong patients' life or even cure the patients at early stages of lung cancer, one of top killer diseases. Therefore, designing comparative trials for the accuracy of scan imaging techniques is rather important at this point, and our nonparametric estimator provides a robust and efficient way to sequentially compare techniques in their staging ability. Other examples may be found on clinincaltrials.gov, which is a website developed by the National Institutes of Health and the Food and Drug Administration to provide information for federally and privately supported clinical trials. One ongoing trial is titled as "Comparison of Cardiac Computed Tomographic 
Angiography (CTA) to Tc-99m Single Photon Emission Computed Tomography (SPECT)" with the purpose of comparing the accuracy of CT and SPECT. In this trial, diagnostic tests are expensive, and the patients' disease status are obtained before tests. These are good logistics for implementing our sequential methods in this trial.

As a final note, in diagnostic imaging trials the results are usually immediately available. The patients' disease status are obtained before tests or shortly after tests. These provide good logistics for conducting sequential diagnostic trials. However, sometimes in biomarker studies it may take a long time to verify true disease status. Also, one may want to look at Youden index or find "optimal" thresholds in addition to comparing diagnostic tests. It will be our future topics to develop proper sequential designs for these issues.

\section{Supplementary Materials}

Web Appendix is available under the Paper Information link at the Biometrics website http://www.biometrics.tibs.org.

\section{Acknowledgement}

The authors would like to thank an associate editor and two referees for their constructive comments and suggestions. This work is supported by NIH grant R01EB005829. This report presents the findings and conclusions of the authors. It does not necessarily represent those of VA HSR\&D Service.

\section{References}

Cai, T. and Pepe, M. S. (2002). Semi-parametric ROC analysis to evaluate biomarkers for disease. Journal of the American Statistical Association 97, 1099-1107.

Dorfman, D. D. and Alf, E. (1969). Maximum-likelihood estimation of parameters of signal- 
detection theory and determination of confidence intervals-rating method data. Journal of Mathematical Psychology. 6, 487-496.

EaSt. Cytel Software Corporation. Cambridge, Massachusetts.

Emerson S., Kittelson J. \& Gillen D. (2007a) Frequentist evaluation of group sequential clinical trial designs. Statistics in Medicine. In press.

Emerson S., Kittelson J. \& Gillen D. (2007b) .On the use of stochastic curtailment in group sequential clinical trials. Statistics in Medicine. 26, 1431-1449.

Gumbel, E. J. (1960). Bivariate exponential distributions. Journal of the American Statistical Association. 55, 698-707.

Hanley, J.A. and McNeil, B.J. (1982). The meaning and use of the area under a receiver operating characteristic (ROC) curve. Radiology. 143, 29-36.

Hanley, J. A. and McNeil, B. J. (1983). A method of comparing the areas under receiver operating characteristic curves derived from the same cases. Radiology. 148, 839-843.

Jennison, C. and Turnbull, B.W. (2000). Group Sequential Methods with Applications to Clinical Trials. Chapman and Hall: New York.

Kim, K. and DeMets, D.L. (1992). Sample size determination for group sequential clinical trials with immediate response. Statistics in Medicine. 11, 1391-1399.

Lan, K. K. G. and DeMets, D. L. (1983). Discrete sequential boundaries for clinical trials. Biometrika. 70, 659-663.

Lardinois, D., Walter Weder, W., Hany, T.F., Kamel, E.M., Korom, S., Seifert, B., von Schulthess, G.K. and Steinert, H.C. (2003). Staging of nonsmall-cell lung cancer with integrated positron-emission tomography and computed tomography. New England Journal of Medicine. 348, 2500-2507.

Mazumdar, M. and Liu A. (2003). Group sequential design for diagnostic accuracy studies. Statistics in Medicine. 22, 727-739. 
Mazumdar, M. (2004). Group Sequential Design for Comparative Diagnostic Accuracy Studies: Implications and Guidelines for Practitioners. Medical Decision Making. 24, 525-533.

Molodianovitch, K., Faraggi, D. and Reiser, B. (2006). Comparing the areas under two correlated ROC curves: parametric and non-parametric approaches. Biometrical Journal. $48,745-57$.

PEST. Medical and Pharmaceutical Statistics Research Unit. University of Reading: Reading, U.K.

S+SeqTrial. Insightful Corporation. Seattle, Washington.

Sanchez-Marin, F. J., Padilla-Medina, J. A. (2006) Alternative performance index to analyze receiver operating characteristic data under the exponential assumption. Journal of Electronic Imaging. 15, Art. No. 023009.

Silvestri, G. A., Tanoue, L. T., Margolis, M. L., Barker, J. and Detterbeck, F. (2003). Noninvasive staging of non-small cell lung cancer. Chest. 123, 137S-146S.

Van der Vaart, A. and Wellner, J. (1996). Weak Convergence and Empirical Processes: With Applications to Statistics. Springer-Verlag, New York.

Wei, L. J., Lin, D. Y. and Weissfeld, L. (1989). Regression analysis of multivariate incomplete failure time databy modeling marginal distributions. Journal of the American Statistical Association. 84, 1065-1073.

Wieand, S., Gail, M. H., James, B. R. and James, K. L. (1989). A family of non-parametric statistics for comparing diagnostic markers with paired or unpaired data. Biometrika. 76, 585-592.

Zhou, X. H., McClish, D. K. and Obuchowski, N. A. (2002). Statistical Methods in Diagnostic Medicine. New York: Wiley.

Zhou, X. H., Sierra M. L., Constantine, A. G. Wilcoxon-based group sequential designs for comparison of areas under two correlated ROC curves. Statistics in Medicine. In press. 
Table 1: Type I error with the nominal level $\alpha=0.05$ in the group sequential designs

\begin{tabular}{|c|c|c|c|c|c|c|c|c|c|c|}
\hline & \multirow{2}{*}{$\frac{\mathrm{m}}{(\mathrm{n})}$} & \multicolumn{3}{|c|}{ WGJJ statistic } & \multicolumn{3}{|c|}{ Parametric } & \multicolumn{3}{|c|}{ Semiparametric } \\
\hline & & Binorm & Bilog & Biexp & Binorm & Bilog & Biexp & Binorm & Bilog & Biexp \\
\hline \multicolumn{11}{|c|}{ Fixed sample design $(J=1)$} \\
\hline & 50 & $6.9 \%$ & $6.6 \%$ & $5.6 \%$ & $4.7 \%$ & $12.0 \%$ & $0.8 \%$ & $31.5 \%$ & $30.4 \%$ & $5.5 \%$ \\
\hline & 100 & $4.5 \%$ & $6.0 \%$ & $6.2 \%$ & $4.1 \%$ & $21.6 \%$ & $1.4 \%$ & $81.2 \%$ & $80.7 \%$ & $5.6 \%$ \\
\hline & 200 & $5.6 \%$ & $6.4 \%$ & $4.0 \%$ & $4.0 \%$ & $37.5 \%$ & $0.8 \%$ & $88.6 \%$ & $90.6 \%$ & $5.5 \%$ \\
\hline \multicolumn{11}{|c|}{ Three-group sequential design $(J=3)$} \\
\hline \multirow[t]{3}{*}{$\mathrm{OBF}$} & 50 & $4.3 \%$ & $5.2 \%$ & $5.2 \%$ & $5.0 \%$ & $30.1 \%$ & $1.0 \%$ & $77.0 \%$ & $75.8 \%$ & $6.6 \%$ \\
\hline & 100 & $4.2 \%$ & $3.3 \%$ & $3.7 \%$ & $2.3 \%$ & $43.8 \%$ & $0.4 \%$ & $98.3 \%$ & $97.4 \%$ & $6.1 \%$ \\
\hline & 200 & $4.7 \%$ & $5.9 \%$ & $6.4 \%$ & $4.5 \%$ & $62.9 \%$ & $1.5 \%$ & $100.0 \%$ & $100.0 \%$ & $5.3 \%$ \\
\hline \multirow[t]{3}{*}{ POC } & 50 & $5.5 \%$ & $6.7 \%$ & $5.7 \%$ & $4.4 \%$ & $26.1 \%$ & $0.8 \%$ & $69.7 \%$ & $66.8 \%$ & $6.8 \%$ \\
\hline & 100 & $5.3 \%$ & $4.5 \%$ & $5.0 \%$ & $3.3 \%$ & $41.4 \%$ & $0.3 \%$ & $96.5 \%$ & $95.5 \%$ & $6.5 \%$ \\
\hline & 200 & $4.7 \%$ & $6.0 \%$ & $6.5 \%$ & $4.1 \%$ & $60.6 \%$ & $1.3 \%$ & $100.0 \%$ & $100.0 \%$ & $5.0 \%$ \\
\hline \multicolumn{11}{|c|}{ Four-group sequential design $(J=4)$} \\
\hline \multirow[t]{3}{*}{$\mathrm{OBF}$} & 50 & $4.7 \%$ & $3.6 \%$ & $5.3 \%$ & $4.2 \%$ & $33.8 \%$ & $0.4 \%$ & $92.5 \%$ & $92.8 \%$ & $6.6 \%$ \\
\hline & 100 & $4.3 \%$ & $4.6 \%$ & $5.2 \%$ & $3.9 \%$ & $52.4 \%$ & $1.0 \%$ & $100.0 \%$ & $99.6 \%$ & $6.5 \%$ \\
\hline & 200 & $5.8 \%$ & $4.8 \%$ & $5.4 \%$ & $4.1 \%$ & $72.6 \%$ & $0.3 \%$ & $100.0 \%$ & $100.0 \%$ & $4.9 \%$ \\
\hline \multirow[t]{3}{*}{ POC } & 50 & $4.5 \%$ & $6.2 \%$ & $6.0 \%$ & $3.4 \%$ & $30.8 \%$ & $0.7 \%$ & $88.7 \%$ & $87.6 \%$ & $6.2 \%$ \\
\hline & 100 & $5.4 \%$ & $3.6 \%$ & $5.8 \%$ & $4.9 \%$ & $48.0 \%$ & $1.2 \%$ & $99.9 \%$ & $99.0 \%$ & $6.1 \%$ \\
\hline & 200 & $4.8 \%$ & $5.1 \%$ & $5.7 \%$ & $3.8 \%$ & $69.4 \%$ & $0.5 \%$ & $100.0 \%$ & $100.0 \%$ & $5.3 \%$ \\
\hline \multicolumn{11}{|c|}{ Five-group sequential design $(J=5)$} \\
\hline \multirow[t]{3}{*}{ OBF } & 50 & $4.9 \%$ & $4.5 \%$ & $5.8 \%$ & $3.9 \%$ & $40.8 \%$ & $0.5 \%$ & $97.4 \%$ & $97.4 \%$ & $5.7 \%$ \\
\hline & 100 & $4.4 \%$ & $5.6 \%$ & $4.7 \%$ & $5.1 \%$ & $60.4 \%$ & $0.6 \%$ & $100.0 \%$ & $99.8 \%$ & $5.3 \%$ \\
\hline & 200 & $4.5 \%$ & $4.5 \%$ & $5.7 \%$ & $3.5 \%$ & $78.8 \%$ & $0.4 \%$ & $100.0 \%$ & $100.0 \%$ & $5.0 \%$ \\
\hline \multirow[t]{3}{*}{ POC } & 50 & $5.9 \%$ & $5.2 \%$ & $6.0 \%$ & $4.1 \%$ & $35.5 \%$ & $0.7 \%$ & $95.2 \%$ & $94.7 \%$ & $5.3 \%$ \\
\hline & 100 & $4.4 \%$ & $5.9 \%$ & $5.1 \%$ & $3.1 \%$ & $55.6 \%$ & $1.2 \%$ & $100.0 \%$ & $99.8 \%$ & $5.0 \%$ \\
\hline & 200 & $5.4 \%$ & $5.4 \%$ & $6.0 \%$ & $3.8 \%$ & $75.9 \%$ & $1.4 \%$ & $100.0 \%$ & $100.0 \%$ & $5.0 \%$ \\
\hline
\end{tabular}

The rejection rate with 1000 realizations. The $95 \%$ prediction interval is $(5.0 \% \pm 1.4 \%)$. 
Table 2: Maximum possible number of subjects in both arms for testing $\Delta^{A}$ or $\Delta^{p A}$ in threegroup analysis (and fixed sample analysis) with $1-\beta=0.80$ and $\alpha=0.05$ under the bivariate normal distribution

\begin{tabular}{|c|c|c|c|c|c|c|}
\hline \multicolumn{7}{|c|}{$\Delta^{A}=\Omega_{1}^{A}-\Omega_{2}^{A}$} \\
\hline$\Omega_{1}^{A} \backslash \Omega_{2}^{A}$ & 0.750 & 0.800 & 0.850 & 0.900 & 0.950 & 0.975 \\
\hline 0.700 & $929(832)$ & 217 (195) & $91(81)$ & $49(44)$ & $32(29)$ & $28(25)$ \\
\hline 0.750 & NA & $793(710)$ & $182(163)$ & $76(68)$ & $43(38)$ & $36(32)$ \\
\hline 0.800 & & NA & $634(568)$ & 143 (128) & $62(55)$ & $48(43)$ \\
\hline 0.850 & & & NA & $456(408)$ & $103(92)$ & $68(61)$ \\
\hline 0.900 & & & & NA & $267(239)$ & $116(103)$ \\
\hline 0.950 & & & & & NA & $416(372)$ \\
\hline \multicolumn{7}{|c|}{$\Delta^{p A}=\Omega_{1}^{p A}-\Omega_{2}^{p A}$} \\
\hline$\Omega_{1}^{p A} \backslash \Omega_{2}^{p A}$ & 0.350 & 0.400 & 0.450 & 0.500 & 0.550 & 0.575 \\
\hline 0.300 & $732(655)$ & $174(156)$ & $72(65)$ & $38(34)$ & $24(21)$ & $20(18)$ \\
\hline 0.350 & NA & $675(604)$ & $156(140)$ & $64(57)$ & $35(31)$ & $28(25)$ \\
\hline 0.400 & & $\mathrm{NA}$ & $573(513)$ & $129(116)$ & $54(48)$ & $41(36)$ \\
\hline 0.450 & & & $\mathrm{NA}$ & $436(390)$ & $97(87)$ & $62(56)$ \\
\hline 0.500 & & & & NA & $273(244)$ & 115 (103) \\
\hline 0.550 & & & & & NA & $505(452)$ \\
\hline
\end{tabular}


Table 3: Maximum possible number of subjects in both arms for testing $\Delta^{A}$ or $\Delta^{p A}$ in threegroup analysis (and fixed sample analysis) with power $1-\beta=0.80$ and $\alpha=0.05$ under the bivariate exponential distribution

\begin{tabular}{|c|c|c|c|c|c|c|}
\hline \multicolumn{7}{|c|}{$\Delta^{A}=\Omega_{1}^{A}-\Omega_{2}^{A}$} \\
\hline$\Omega_{1}^{A} \backslash \Omega_{2}^{A}$ & 0.750 & 0.800 & 0.850 & 0.900 & 0.950 & 0.975 \\
\hline 0.700 & $1244(1113)$ & $293(262)$ & $122(109)$ & $65(58)$ & $39(35)$ & $32(28)$ \\
\hline 0.750 & NA & $1094(979)$ & $253(226)$ & $104(93)$ & $54(49)$ & $42(37)$ \\
\hline 0.800 & & NA & $914(818)$ & $206(184)$ & $83(74)$ & $58(52)$ \\
\hline 0.850 & & & NA & $705(631)$ & $152(136)$ & $90(80)$ \\
\hline 0.900 & & & & NA & $461(412)$ & $180(161)$ \\
\hline 0.950 & & & & & NA & $975(872)$ \\
\hline \multicolumn{7}{|c|}{$\Delta^{p A}=\Omega_{1}^{p A}-\Omega_{2}^{p A}$} \\
\hline$\Omega_{1}^{p A} \backslash \Omega_{2}^{p A}$ & 0.350 & 0.400 & 0.450 & 0.500 & 0.550 & 0.575 \\
\hline 0.300 & $852(763)$ & $203(182)$ & $84(76)$ & $44(39)$ & $26(24)$ & $21(19)$ \\
\hline 0.350 & NA & $785(703)$ & $183(164)$ & $75(67)$ & $39(35)$ & $30(27)$ \\
\hline 0.400 & & NA & $680(609)$ & $154(138)$ & $62(55)$ & $43(39)$ \\
\hline 0.450 & & & NA & $539(482)$ & 117 (104) & $69(62)$ \\
\hline 0.500 & & & & NA & $362(324)$ & $142(127)$ \\
\hline 0.550 & & & & & NA & 781 (699) \\
\hline
\end{tabular}

